(Although admittedly, sophisticated computer-aided searches can now help scientists sift through the massive volume of published literature.)

The times between submission and publication for the articles in the 1990 issues will not be attractive to potential contributors, being long (10-12 months) compared with those of leading international journals, such as Perkin Transactions (4-6 months); but I guess this situation will improve if the journal becomes more popular, and as the refereeing, editing and printing of the papers becomes commonplace.

The journal will be a useful vehicle for scientists working with polycyclic aromatic hydrocarbons. It would also be of interest to chemists and biologists working in fields that are often relevant to these compounds, although I doubt whether many of these researchers will get around to reading it.

Stanley Roberts is in the Department of Chemistry, University of Exeter, Exeter EX4 4QD, UK.

\section{Great shakes}

\section{Derek Steele}

Vibrational Spectroscopy: A Section of Analytica Chimica Acta. Editors J. G. Grasselli and J. H. van der Maas. Elsevier. 4/yr. Dfl. 332.

THE declared aim of this new journal is to "assemble the research findings in the field of vibrational spectroscopy, now scattered through the literature, in a single journal." There are, however, several journals already in existence devoted to this subject (Spectrochimica Acta, Journal of Molecular Structure, Journal of Molecular Spectroscopy and so on). So it is difficult to see how the publication of this new journal can be justified. Nevertheless, journals continue to proliferate despite library-funding crises. Accepting this inevitability although not its logic, what has Vibrational Spectroscopy got in its favour?

The editors are spectroscopists of high calibre and can be relied upon to maintain a high standard of refereeing and editing. Indeed, several well-written articles in the initial issues describe important new developments. Most papers are on analytical aspects of spectroscopy, as is proper for a journal which is an off-shoot of Analytica Chimica Acta. Also, there are some good theoretical papers - witness the article by Gantz et al. entitled 'Continium resonance Raman scattering in diatomic molecules'.

Articles on Raman spectroscopy appear as frequently as those on infrared spectroscopy, and there are many papers on technique developments. Depthprofiling, Langmuir-Blodgett films, photoacoustic techniques and microprobe investigations of electrodes are all covered in the first four issues.

In addition to these research publications, some excellent review articles are included, such as H. J. Luinge's review in the first issue of automated interpretation of vibrational spectra.

Half of the second and all of the third issues consist of selected papers that have arisen from conferences. With so many journals in competition for good material it is inevitable that this means of acquiring copy should be prevalent. It is, however, somewhat undesirable in that conference proceedings more often than not consist of material that is being published elsewhere. Having said that, I confess that I found such papers presented here interesting and of good quality.

The journal has got off to a good start and I wish it well. It would have been nice, however, if its birth was accompanied by the demise of a journal elsewhere. Unfortunately, such idealism does not seem to have a place in this practical world.

Derek Steele is in the Department of Chemistry, Royal Holloway and Bedford New College, University of London, Egham Hill, Egham, Surrey TW2O OEX, UK.

\section{Seamless joins}

\section{Michael J. Gait}

Bioconjugate Chemistry. Editor-in-chief Claude F. Meares. American Chemical Society. 6/yr. US \$249, Canada and Mexico \$257, Europe \$266, elsewhere $\$ 273$ (nonmembers); US $\$ 29$, Canada $\$ 37$, elsewhere $\$ 46$ (members).

"BIOCONJUGATE Chemistry addresses the joining of two different molecular functions by chemical or biological means". This quote from the journal's advertising blurb prompts the immediate reaction: "Why do we need a journal for this?"

The answer is quickly found by browsing through a few recent issues. First, many different chemical and biological subjects are represented; before this journal came into being in 1990, the topics represented were scattered throughout many disparate journals. More importantly, the journal has an immediate and wide-ranging appeal. This is partly due to the basic fascination of observing what happens when two dissimilar molecules are joined together: the result is not always the sum of the parts. But the unifying theme is hinted at by the prefix 'Bio'. Beyond the mere joining of two molecular species, there is a clear and overriding aim to generate novel and useful combinations of biological and chemical properties within a single entity. This sense of purpose nicely ties in the various disciplines and provides a tangible justification for the journal's existence.

Some topics are more highly represented than others, notably immunoconjugates. But there is a healthy spread of papers involving proteins, polypeptides, oligonucleotides, oligosaccharides, drugs and other biomolecules. The juxtaposition of these topics is enjoyable, because one is exposed to a wide variety of reagents and techniques from different fields. So the journal provides a focus for the interchange of ideas and techniques between disciplines.

For a fledgling journal, there are many solid and reassuring aspects of style. Both text and figures are easy to read and occasional issues carry excellent full-colour reproductions. $\mathrm{Ex}$ perimental procedures are rightly featured before the results and discussion sections, and there is the usual high standard of chemical characterization expected of a journal from the American Chemical Society.

Apart from articles and reviews, there are letters (essentially short articles of less than 1,000 words) and, unusually, an occasional teaching editorial, which will be helpful to the many nonspecialists who are likely to read the journal. There is no doubt that it will occupy an important niche on library and personal bookshelves for many years to come.

Michael J. Gait is in the Medical Research Council Laboratory of Molecular Biology, Hills Road, Cambridge CB2 $2 Q H$, UK.

\section{Fostering interactions}

\author{
Michael G. B. Drew
}

Structural Chemistry. Editors István Hargittai and Arthur Greenberg. $\mathrm{VCH}$. 6/yr. \$295, £195, DM 607 (institutional); \$95, £78, DM 210 (personal).

THE aim of this journal is to encourage a broad spectrum of articles under the general title of structural chemistry and hence to foster interaction between branches of structural chemistry. Structural chemistry is defined to include experimental structure determination, experimental thermochemistry, and other relevant techniques such as photoelectron spectroscopy, as well as computational studies, new structural models and bonding theories.

These aims have been well achieved in 